\title{
Applications of Parameterized st-Orientations in Graph Drawing Algorithms
}

\author{
Charalampos Papamanthou ${ }^{1,2}$ and Ioannis G. Tollis ${ }^{1,2}$ \\ 1 Department of Computer Science, University of Crete, P.O. Box 2208, \\ Heraklion, Greece \\ \{cpap, tollis\}@csd.uoc.gr \\ 2 Institute of Computer Science, FORTH, Vasilika Vouton, P.O. Box 1385, \\ Heraklion, GR-71110, Greece \\ \{cpap, tollis\}@ics.forth.gr
}

\begin{abstract}
Many graph drawing algorithms use st-numberings (st-orientations or bipolar orientations) as a first step. An st-numbering of a biconnected undirected graph defines a directed graph with no cycles, one single source $s$ and one single sink $t$. As there exist exponentially many st-numberings that correspond to a certain undirected graph $G$, using different $s t$-numberings in various graph drawing algorithms can result in aesthetically different drawings with different area bounds. In this paper, we present results concerning new algorithms for parameterized st-orientations, their impact on graph drawing algorithms and especially in visibility representations.
\end{abstract}

\section{Introduction}

st-orientations (st-numberings) or bipolar orientations are orientations of undirected graphs that satisfy some certain criteria, i.e., they define no cycles and have exactly one source $s$ and one sink $t$. Starting with an undirected biconnected graph $G=(V, E)$, many graph drawing algorithms, such as hierarchical drawings [1], visibility representations [2] and orthogonal drawings [3], use an storientation of $G$ in order to compute a drawing of $G$. Therefore, the importance of st-orientations in Graph Drawing is evident.

Given a biconnected undirected graph $G=(V, E)$, with $n$ vertices and $m$ edges, and two nodes $s, t$, an $s t$-orientation (also known as bipolar orientation or st-numbering) of $G$ is defined as an orientation of its edges such that a directed acyclic graph with exactly one source $s$ and exactly one sink $t$ is produced. An st-orientation of an undirected graph can be easily computed using an stnumbering [4] of the respective graph $G$ and orienting the edges of $G$ from low to high. An st-numbering of $G$ is a numbering of its vertices such that $s$ receives number $1, t$ receives number $n$ and every other node except for $s, t$ is adjacent to at least one lower-numbered and at least one higher-numbered node.

st-numberings were first introduced in 1967 in [5], where it is proved (together with an $O(n m)$ time algorithm) that given any edge $\{s, t\}$ of a biconnected undirected graph $G$, we can define an st-numbering. However, in 1976 Even and 
Tarjan proposed an algorithm that computes an st-numbering of an undirected biconnected graph in $O(n+m)$ time [4]. Ebert [6] presented a slightly simpler algorithm for the computation of such a numbering, which was further simplified by Tarjan [7]. The planar case has been extensively investigated in [8] where a linear time algorithm is presented which may reach any st-orientation of a planar graph. Finally, in [9] a parallel algorithm is described. An overview of the work concerning bipolar orientations is presented in [10].

However, all developed algorithms compute an st-numbering at random, without expecting any specific properties of the oriented graph. In this paper we present new techniques that produce such orientations with specific properties. Namely, our techniques are able to control the length of the longest path of the resulting directed acyclic graph. This provides significant flexibility to many graph drawing algorithms such as [2,3. Actually, st-orientations play a very important role in defining certain aesthetics in the drawings produced by algorithms they use them. The length of the longest path of the final directed graph that is produced is vital in determining the area bounds of the drawing. In this paper, we try to answer these questions by connecting a newly developed algorithm for the computation of st-orientations with graph drawing applications.

The paper is organized as follows. In Section 2 we present the problem, the objectives and some preliminary definitions. In Section 3 we give a brief description of the algorithm and show its implication in defining the longest path length of the final directed graph. A detailed presentation of the algorithm can be found in 11]. In Section 4 we comment on primal and dual st-orientations and Section 5 presents experimental results. Finally, some conclusions are presented in Section 6.

\section{Preliminaries}

\subsection{Motivation and Objectives}

Many algorithms in Graph Drawing use st-Orientations as a first step. Additionally, the length of the longest path from $s$ to $t$ of the specific st-orientation determines certain aesthetics of the drawing:

- Hierarchical Drawings. One of the most common algorithms in hierarchical drawing is the longest path layering [1. This algorithm applies to directed acyclic graphs. The height of such a drawing is always equal to the length of the longest path of the directed acyclic graph, $l$. If we want to visualize an undirected graph $G$ using this algorithm, we must firstly st-orient $G$. The height of the produced drawing will be equal to the length of the longest path $l$ of the produced st-orientation.

- Visibility Representations. In order to compute visibility representations of planar graphs, we must compute an optimal topological numbering of an st-orientation of the input graph [2]. This can be done if we assign unitweights to the edges of the graph and compute the longest path to each one of its vertices from source $s$. The $y$-coordinate of each vertex $u$ in the 
visibility representation is equal to the length of the longest path from $s$ to $u$. Hence the length of the longest path of the used st-orientation is decisive in visibility representations of undirected graphs. Moreover, in the visibility representations, the length of the longest path of the dual graph is also important. How a different primal st-orientation impacts on the dual orientation is very crucial for visibility representations.

- Orthogonal Drawings. The first step of algorithms that compute orthogonal drawings [3] is to compute an st-numbering of the input undirected graph $G$. These algorithms compute some variables (such as the row pairs or the column pairs in [3]) that are functions of the st-orientation and which determine the width and the height of the drawing. Applying different storientations for the orthogonal drawing of a graph $G$, can result in different drawing area bounds.

Figure 1 depicts an undirected graph $G$ (Figure 1 a) and two different storientations of it. Figure 2 shows two different longest path and visibility representation layouts for the two different st-orientations (1) b), (1k) of the same graph (1a). Note that the drawings have different characteristics, which depend on the length of the longest path of the different st-orientations.

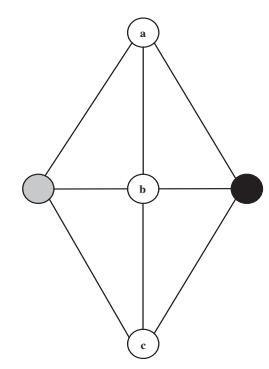

(a)

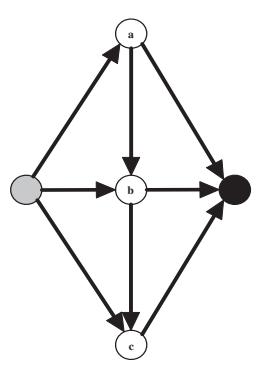

(b)

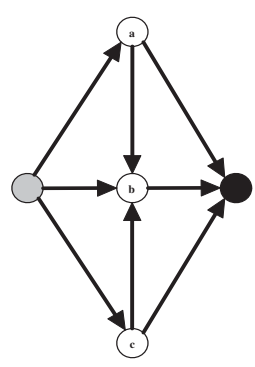

(c)

Fig. 1. An undirected graph (a) and two (b), (c) possible st-orientations of it

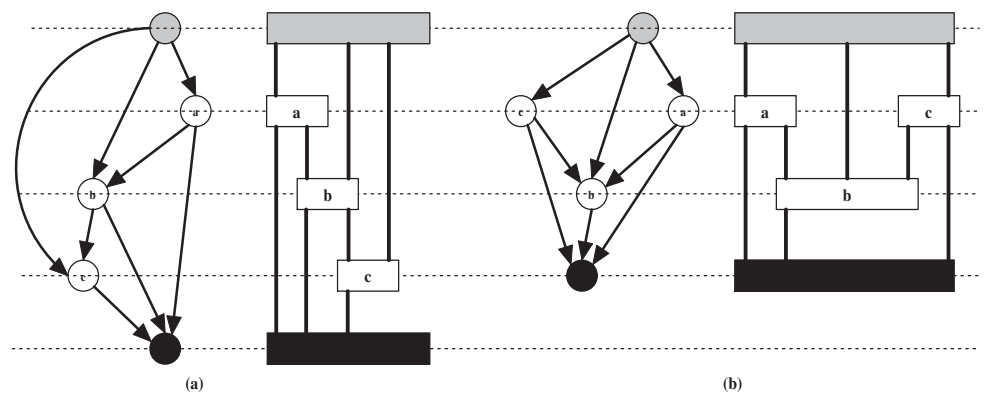

Fig. 2. Longest path layering and visibility representation layouts for the $s$-orientation of Figure 1b (a) and for this of Figure 15 (b) 
In order to develop an algorithm for the computation of (longest-path) parameterized st-orientations, there are mainly two things that we should carefully consider: (1), the correctness of the final st-orientation and (2), the algorithm should give us the opportunity to control the length of the longest path of the final directed graph. The idea behind the algorithm is that, beginning with an undirected biconnected graph $G$ and two nodes of it $s, t$, we repeatedly remove a node $v_{i}$ (different from $t$ ), orienting at the same time all its incident edges from $v_{i}$ to its neighbors. In this way we build up a directed graph $F$. The first node removed is the source $s$, of the desired st-orientation. Thus, the problem of computing a correct st-orientation is reduced to this of removing the vertices of the graph with a correct order $v_{1}, v_{2}, \ldots, v_{n}$ with $v_{1}=s$ and $v_{n}=t$ and simultaneously maintaining a data structure that will allow us to compute such a correct order.

\subsection{Terminology}

In this section, we present some terminology and useful observations. Throughout the paper, $N_{G}(v)$ denotes the set of neighbors of node $v$ in graph $G, s$ the source and $t$ the sink of the graph. Additionally, $l$ is the length of the longest path of the primal graph from $s$ to $t$, whereas $l^{*}$ denotes the length of the longest path of the respective dual graph. Let $G=(V, E)$ be a one-connected undirected graph, i.e., a graph that contains at least one vertex whose removal causes the initial graph to disconnect and $T=(B \cup C, U)$ be the respective block-cutpoint tree [12. The edges $(i, j) \in U$ of the block-cutpoint tree always connect pairs of blocks (biconnected components) and cutpoints such that the cutpoint of a tree edge belongs to the vertex set of the corresponding block (see Figure 3).
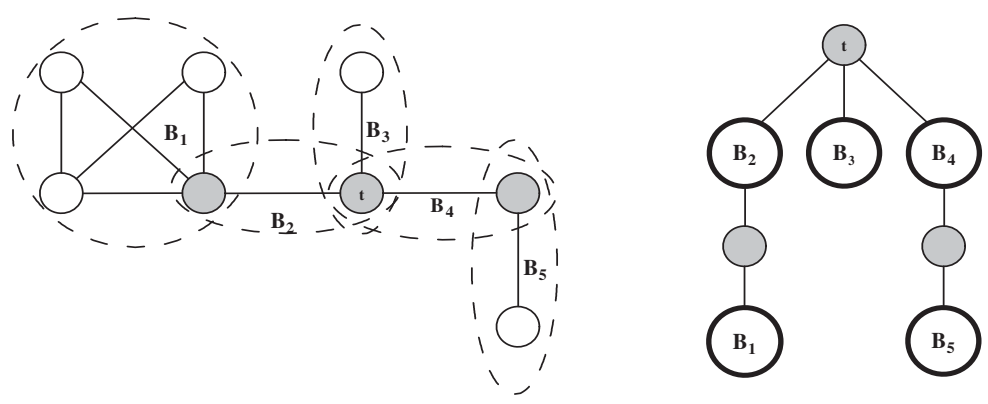

Fig. 3. A one-connected graph and the $t$-rooted block-cutpoint tree

The block-cutpoint tree is a free tree, i.e., it has no distinct root. In order to transform this free tree into a rooted tree, we define the $t$-rooted block-cutpoint tree with respect to the sink $t$. Consequently, the root of the block-cutpoint tree is the block that contains $t$ (see Figure 33).

Finally, we define the leaf-blocks of the $t$-rooted block-cutpoint tree to be the blocks, except for the root of the block-cutpoint tree that contain a single 
cutpoint. The block-cutpoint tree can be computed in $O(n+m)$ time with an algorithm similar to DFS [12].

Following, we give some results that are necessary for the development of the algorithm.

Lemma 1 ([11]). Let $G=(V, E)$ be an undirected biconnected graph and $s, t$ be two of its nodes. Suppose we remove $s$ and all its incident edges. Then there is at least one neighbor of s lying in a leaf-block of the t-rooted block-cutpoint tree. Moreover, this neighbor is not cutpoint.

The main idea of the algorithm is based on the successive removal of nodes and the simultaneous update of the $t$-rooted block-cutpoint tree. We call each such node a source, because at the time of its removal it is effectively chosen to be a source of the remainder of the graph. We initially remove $s$, the first source, which is the source of the desired st-orientation and give direction to all its incident edges from $s$ to all its neighbors. After this removal, the graph either remains biconnected or is decomposed into several biconnected components but the number of leaf-blocks remains the same or is decomposed into several biconnected components and the number of leaf-blocks changes.

This procedure continues until all nodes of the graph but one are removed. As it will be clarified in the next sections, at every step of the algorithm there will be a set of potential sources to choose from. Our aim is to establish a connection between the current source choice and the length of the longest path of the produced $s$-oriented graph.

\section{Parameterized st-Orientations}

\subsection{The Algorithm}

Now we describe the procedure in a more formal way. We name this procedure STN. Let $G=(V, E)$ be an undirected biconnected graph and $s, t$ two of its nodes. We will compute an st-orientation of $G$. Suppose we recursively produce the graphs $G_{i+1}=G_{i}-\left\{v_{i}\right\}$, where $v_{1}=s$ and $G_{1}=G$ for all $i=1, \ldots, n-1$.

During the procedure we always maintain a $t$-rooted block-cutpoint tree. Additionally, we maintain a structure $Q$ that plays a major role in the choice of the current source. $Q$ initially contains the desired source for the final orientation, $s$. Finally we maintain the leaf-blocks of the $t$-rooted block-cutpoint tree. During every iteration $i$ of the algorithm node $v_{i}$ is chosen so that

- it is a non-cutpoint node that belongs to $Q(1)$

- it belongs to a leaf-block of the $t$-rooted block-cutpoint tree (2)

Note that for $i=1$ there is a single leaf-block (the initial biconnected graph) and the cutpoint that defines it is the desired sink of the orientation, $t$. When a source $v_{i}$ is removed from the graph, we have to update $Q$ in order to be able to choose our next source. $Q$ is then updated by removing $v_{i}$ and by inserting all of the neighbors of $v_{i}$ except for $t$. 
By Lemma 1, after the removal of a node $v_{i}$, there will always exist at least one node satisfying both (1) and (2). In this way we can reach the final sink of the orientation, $t$, without disconnecting the graph. Additionally, each time a node $v_{i}$ is removed we orient all its incident edges from $v_{i}$ to its neighbors. The procedure continues until $Q$ gets empty. Let $F=\left(V^{\prime}, E^{\prime}\right)$ be the directed graph computed by this procedure. We claim [1] that $F=\left(V^{\prime}, E^{\prime}\right)$ is an st-oriented graph:

Theorem 2 ([11]). The directed graph $F=\left(V^{\prime}, E^{\prime}\right)$ computed by $S T N$ is storiented.

STN is a recursive algorithm for computing an st-orientation of a biconnected undirected graph $G$. The full pseudocode and an illustrative example can be found in [11. During the execution of the algorithm we can also compute an st-numbering $f$ of the initial graph. Actually, for each node $v_{i}$ that is removed from the graph, the subscript $i$ is the final st-number of node $v_{i}$. Finally, each node $v$ inserted into $Q$ is associated with a timestamp value $m(v)$ (which will finally determine the longest path length). $m(v)$ is set equal to $i$, every time that $v$ is discovered by a removed node $v_{i}$, i.e., $v$ is a neighbor of $v_{i}$. This means that $m(v)$ can be updated many times until the algorithm terminates.

Let us now comment on the execution time of the algorithm. Each time a vertex is removed, we have to update the block-cutpoint tree, which takes time $O(n+m)$ 12. As all the vertices are removed, the algorithm runs clearly in $O(n m)$ time. However we can use the algorithm for biconnectivity maintenance (which supports edge deletions in $O\left(\log ^{5} n\right)$ time) proposed in [13] and drop the bound to $O\left(m \log ^{5} n\right)[11$.

\subsection{Control of the Length of Longest Path}

This section presents methods which can be implemented in order to control the length of the longest path of an st-orientation computed with STN. Actually, we take advantage of the timestamps $m(u)$ in order to choose our next source. During iteration $j$ of the algorithm, we have to pick a leaf-block $B_{j}^{l}$ of the $t$ rooted block-cutpoint tree and we always have to make a choice on the structure $Q^{\prime}=B_{j}^{l} \cap Q \sim\left\{h_{j}^{l}\right\}$, where $h_{j}^{l}$ is the cutpoint that defines $B_{j}^{l}$. Our investigation has revealed that if vertices with high timestamp are chosen then long sequences of vertices are formed and thus there is higher probability to obtain a long longest path. We call this way of choosing vertices MAX-STN. Actually, MAXSTN resembles a DFS traversal (it searches the graph at a maximal depth). Hence, during MAX-STN, the next source $v$ is arbitrarily chosen from the set

$$
\left\{v \in Q^{\prime}: m(v)=\max \left\{m(i): i \in Q^{\prime}\right\}\right\} .
$$

On the contrary, we have observed that if vertices with low timestamp are chosen, then the final st-oriented graph has relatively small longest path. We call this way of choosing vertices MIN-STN, which in turn resembles a BFS traversal. Hence, during MIN-STN, the next source $v$ is arbitrarily chosen from the set

$$
\left\{v \in Q^{\prime}: m(v)=\min \left\{m(i): i \in Q^{\prime}\right\}\right\}
$$


The length of a longest path from $s$ to $t$ computed with MAX-STN is denoted with $\ell(t)$ whereas this computed with MIN-STN is denoted with $\lambda(t)$. As it has already been reported, it would be desirable to be able to compute st-oriented graphs of length of longest path within the interval $[\lambda(t), \ell(t)]$. This is called a parameterized st-orientation. So the question that arises is: Can we insert a parameter into our algorithm, for example a real constant $p \in[0,1]$ so that our algorithm computes an st-oriented graph of length of longest path that is a function of $p$ ?

This is feasible if we modify STN. As the algorithm is executed exactly $n$ times ( $n$ vertices are removed from the graph), we can execute the procedure MAXSTN for the first $p n$ iterations and the procedure MIN-STN for the remaining $(1-p) n$ iterations. We call this method PAR-STN $(p)$ and we say that it produces an $s t$-oriented graph with length of longest path from $s$ to $t$ equal to $\Delta(p)$. Note that PAR-STN $(0)$ is equivalent to MIN-STN, thus $\Delta(0)=\lambda(t)$ while PAR$\operatorname{STN}(1)$ is equivalent to MAX-STN and $\Delta(1)=\ell(t)$. PAR-STN has been tested and it seems that when applied to st-Hamiltonian graphs (biconnected graphs that contain at least one path from $s$ to $t$ that contains all the nodes of the graph) there is a high probability that $\Delta(p) \geq p(n-1)$. Actually, $\Delta(p)$ is very close to $p(n-1)$. Additionally, it has been observed that if we switch the order of MAX-STN and MIN-STN execution, i.e., execute MIN-STN for the first $p n$ iterations and MAX-STN for the remaining $(1-p) n$ iterations, there is a high probability that $\Delta(p) \leq p(n-1)$. In this case, $\Delta(p)$ is again very close to $p(n-1)$.

\section{Primal and Dual st-Orientations}

\subsection{General}

Now we present some results concerning the impact of parameterized st-orientations on st-planar graphs. If we st-orient such a graph, we can define a single orientation for the dual graph $G^{*}$ which is also an $s^{*} t^{*}$-orientation.

This method is used in the visibility representations algorithms [2, when we have to compute the dual $s^{*} t^{*}$-oriented graph. The length of the longest path of this graph determines the width of the geometric representation. Thus, the questions that arise are natural. What is the impact of the parameter $p$ on the length of the longest path of the dual $s^{*} t^{*}$-oriented graph $G^{*}$ of an $s t$ planar graph $G$, which (the graph $G$ ) has been $s t$-oriented with $\operatorname{PAR}-\operatorname{STN}(p)$ ? Intuitively, we would expect that $l^{*}$ (the length of the longest path of the dual graph $G^{*}$ ) will grow inversely proportional to $l$. As we will see, this is not always the case.

\subsection{A Special Class of Planar Graphs}

In this section we investigate certain classes of $s$-planar graphs that can be st-oriented in such a way that certain lengths of primal and dual longest paths can be achieved. This is actually a good reason to justify the fact that different st-orientations are indeed important in many applications. 
Definition 3. We define an n-path planar graph $(n \geq 5) G=(V, E)$ to be the planar graph that consists of a path $P=v_{2}, v_{3}, \ldots, v_{n-1}$ of $n-2$ nodes and two other nodes $v_{1}, v_{n}$ such that $\left(v_{1}, v_{i}\right) \in E,\left(v_{i}, v_{n}\right) \in E \forall i=2, \ldots n-1$ and $\left(v_{1}, v_{n}\right) \in E$.
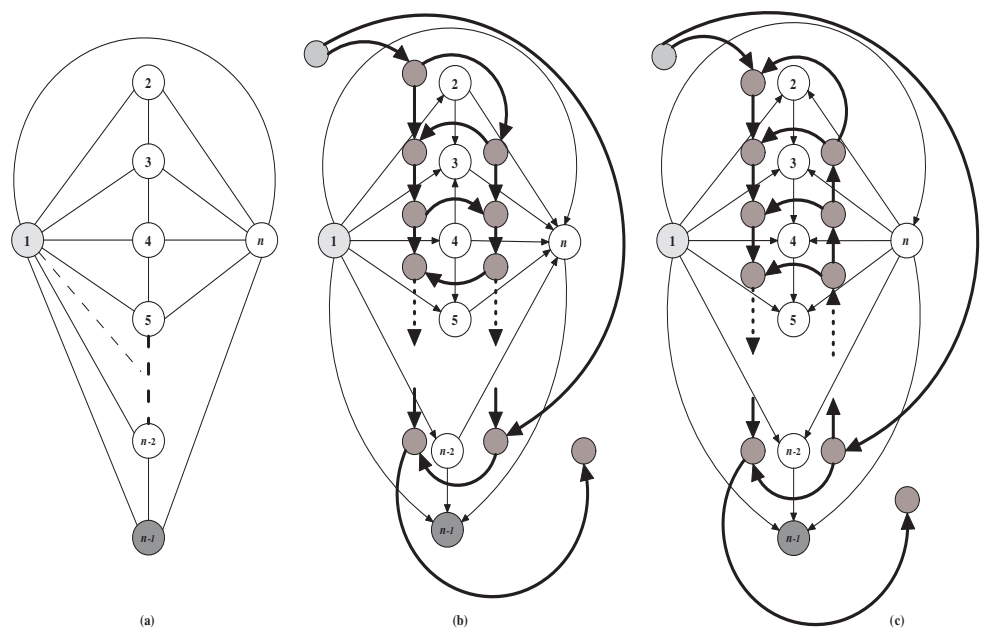

Fig. 4. (a) An $n$-path planar graph. We define node 1 to be the source of the graph and node $n-1$ to be the sink of the graph. (b) Primal and Dual st-orientation with $l=4$ and $l^{*}=2 n-4$. (c) Primal and Dual st-orientation with $l=n-1$ and $l^{*}=2 n-4$.

In Figure $4 a$, one $n$-path planar graph is depicted. Its source is node 1 whereas its sink is node $n-1$. Note that an $(n+1)$-path planar graph $G_{n+1}$ can be obtained from an $n$-path planar graph $G_{n}$ if we add a new node and connect it with nodes $v_{1}, v_{2}$ and $v_{n}$ (nodes $v_{1}$ and $v_{n}$ are the rightmost and leftmost nodes of $G_{n}$ 's embedding in Figure 4). Let now $G_{n}$ be an $n$-path planar graph and $\lambda\left(G_{n}\right), \ell\left(G_{n}\right)$ denote the minimum and the maximum longest path length $1(n-1)$-orientations over the set of all the $1(n-1)$-orientations of $G_{n}$ respectively. In Figure $4 \mathrm{~b}$, the primal orientation of minimum longest path length (together with the respective dual orientation of longest path length $\left.\lambda^{*}\left(G_{n}\right)\right)$ is depicted while in Figure 4. the orientation of maximum longest path length (together with respective dual orientation of longest path length $\left.\ell^{*}\left(G_{n}\right)\right)$ is depicted. Inductively, we can prove that for an $n$-path planar graph the following holds:

Theorem 4. For all $n \geq 5$, it is $\lambda\left(G_{n}\right)=4$, $\ell\left(G_{n}\right)=n-1$ and $\lambda^{*}\left(G_{n}\right)=$ $\ell^{*}\left(G_{n}\right)=2 n-4$.

According to Theorem 4, the impact of different st-orientations of an $n$-path planar graph on the area of their visibility representation is evident. By using the minimum st-orientation, we will need an area equal to

$$
\lambda\left(G_{n}\right) \lambda^{*}\left(G_{n}\right)=4(2 n-4)=8 n-16=O(n)
$$


If we use the maximum st-orientation, we will need an area equal to

$$
\ell\left(G_{n}\right) \ell^{*}\left(G_{n}\right)=(n-1)(2 n-4)=2 n^{2}-6 n+4=O\left(n^{2}\right)
$$

Note that while $\ell\left(G_{n}\right)+\ell^{*}\left(G_{n}\right)=3 n-5>2 n$, it is $\lambda\left(G_{n}\right)+\lambda^{*}\left(G_{n}\right)=2 n \leq 2 n$. We therefore introduce the following conjecture:

Conjecture 5. For every $n$-node planar biconnected graph $G$, two nodes $s, t$ of its vertex set, there exists at least one st-orientation of $G$ such that $l+l^{*} \leq 2 n+c$, where $c$ is a constant.

In order to face this conjecture, one should try to devise an algorithm that deterministically st-orients a planar graph in a way that the produced length of the dual longest path grows at most as much as the primal one does.

\section{Experimental Results}

Following we present our results for different kinds of graphs, st-Hamiltonian graphs (undirected graphs that have at least one Hamilton path from $s$ to $t$ and hence an upper bound for the longest path length equal to $n-1$ ) and planar graphs. All experiments were run on a Pentium IV machine, 512 MB RAM, 2.8 GH under Windows 2000 professional.

\section{1 st-Hamiltonian Graphs}

We have implemented the algorithm in Java, using the Java Data Structures Library (www.jdsl.org) [14. The graphs we have tested are $n$-node-undirected

Table 1. Results for density 3.5 st-Hamiltonian graphs

\begin{tabular}{|c||c|c|c|c|c|c||c||c|c|c|}
\hline \multicolumn{1}{|c||}{$\mathbf{n}$} & $\mathbf{p}$ & $\mathbf{p}=\mathbf{0}$ & \multicolumn{2}{c|}{$\mathbf{p = 0 . 3}$} & \multicolumn{2}{c|}{$\mathbf{p = 0 . 5}$} & \multicolumn{2}{c|}{$\mathbf{p = 0 . 7}$} & \multicolumn{2}{c|}{$\mathbf{p = 1}$} \\
\cline { 2 - 14 } & $l$ & $\%(n-1)$ & $l$ & $\%(n-1)$ & $l$ & $\%(n-1)$ & $l$ & $\%(n-1)$ & $l$ & $\%(n-1)$ \\
\hline 100 & 14.00 & 0.141 & 38.90 & 0.393 & 59.20 & 0.598 & 76.50 & 0.773 & 92.20 & 0.931 \\
\hline 200 & 18.60 & 0.093 & 74.10 & 0.372 & 113.00 & 0.568 & 147.90 & 0.743 & 186.60 & 0.938 \\
\hline 300 & 23.30 & 0.078 & 104.80 & 0.351 & 165.10 & 0.552 & 219.20 & 0.733 & 280.70 & 0.939 \\
\hline 400 & 23.30 & 0.058 & 139.10 & 0.349 & 213.80 & 0.536 & 289.30 & 0.725 & 376.30 & 0.943 \\
\hline 500 & 29.20 & 0.059 & 169.40 & 0.339 & 267.30 & 0.536 & 361.20 & 0.724 & 470.70 & 0.943 \\
\hline 600 & 27.90 & 0.047 & 202.10 & 0.337 & 318.90 & 0.532 & 428.90 & 0.716 & 566.60 & 0.946 \\
\hline 800 & 30.00 & 0.038 & 264.90 & 0.332 & 415.30 & 0.520 & 566.50 & 0.709 & 755.60 & 0.946 \\
\hline 900 & 31.70 & 0.035 & 294.30 & 0.327 & 469.90 & 0.523 & 640.20 & 0.712 & 848.10 & 0.943 \\
\hline 1000 & 36.20 & 0.036 & 322.10 & 0.322 & 518.20 & 0.519 & 709.30 & 0.710 & 940.00 & 0.941 \\
\hline 1100 & 38.90 & 0.035 & 353.90 & 0.322 & 576.30 & 0.524 & 782.90 & 0.712 & 1033.40 & 0.940 \\
\hline 1200 & 34.40 & 0.029 & 387.00 & 0.323 & 622.10 & 0.519 & 845.50 & 0.705 & 1127.80 & 0.941 \\
\hline 1300 & 34.30 & 0.026 & 421.10 & 0.324 & 674.50 & 0.519 & 917.00 & 0.706 & 1223.10 & 0.942 \\
\hline 1400 & 38.90 & 0.028 & 448.80 & 0.321 & 718.40 & 0.514 & 983.90 & 0.703 & 1319.90 & 0.943 \\
\hline 1500 & 38.00 & 0.025 & 478.30 & 0.319 & 775.70 & 0.517 & 1056.40 & 0.705 & 1417.10 & 0.945 \\
\hline 1600 & 39.30 & 0.025 & 515.00 & 0.322 & 824.30 & 0.516 & 1137.20 & 0.711 & 1499.10 & 0.938 \\
\hline 1700 & 38.50 & 0.023 & 539.30 & 0.317 & 872.00 & 0.513 & 1190.40 & 0.701 & 1604.00 & 0.944 \\
\hline 1800 & 41.10 & 0.023 & 571.90 & 0.318 & 923.60 & 0.513 & 1263.80 & 0.703 & 1691.30 & 0.940 \\
\hline 1900 & 41.40 & 0.022 & 605.60 & 0.319 & 978.60 & 0.515 & 1331.80 & 0.701 & 1786.30 & 0.941 \\
\hline 2000 & 44.00 & 0.022 & 632.40 & 0.316 & 1023.80 & 0.512 & 1403.50 & 0.702 & 1883.90 & 0.942 \\
\hline
\end{tabular}


st-Hamiltonian graphs of density $d$ where $n=100,200,300, \ldots, 2000$ and $d=$ 3.5. For each pair $(n, d)$ we have tested 10 different randomly generated graphs (and we present the mean of the length of the longest path) in order to get more reliable results. We have similar results for other values of density as well (see Figure 5].

As we can see, the results (Table 1 and Figure 5) are remarkably consistent with the parameter $p$. The computed longest path length for $p=p_{0}$ is always very close to $p_{0}(n-1)$. The computed results are similar for increasing graphs size and density.

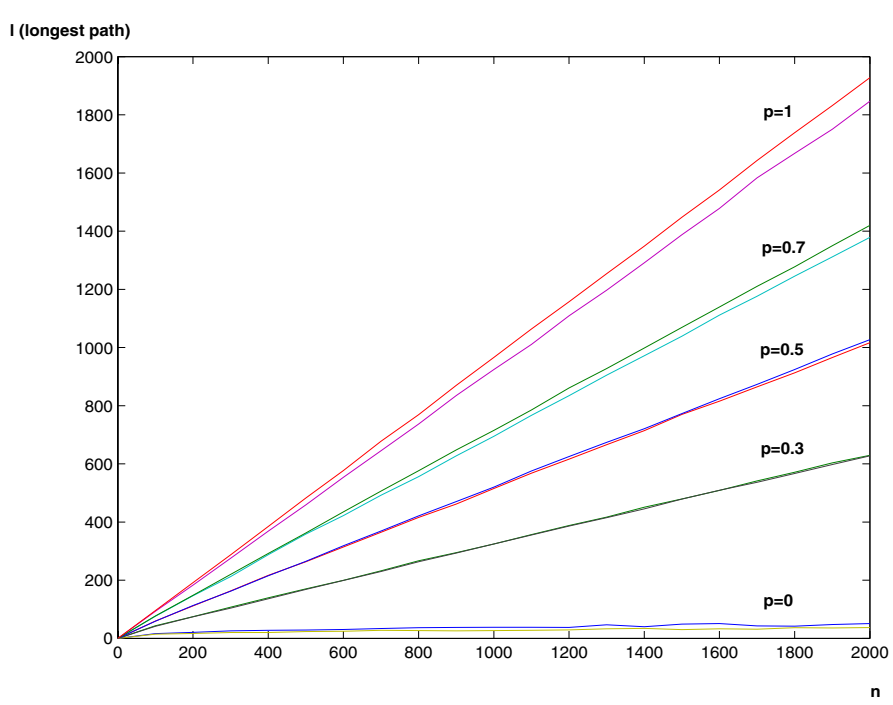

Fig. 5. Longest path length as a function of $n, d, p(d=2.5,6.5)$

Table 2. Primal and dual longest path length for triangulated st-planar graphs

\begin{tabular}{|c|c||c|c|c||c|c|c||c|c|c||c|c|c|}
\hline \multicolumn{9}{|c|}{} & \multicolumn{3}{|c|}{$\mathbf{p = 0}$} & \multicolumn{3}{c|}{$\mathbf{p = 0 . 5}$} & \multicolumn{3}{c|}{$\mathbf{p = 1}$} & \multicolumn{3}{|c|}{$l \times l^{*}$} \\
\hline$n$ & $2 n$ & $l$ & $l^{*}$ & $l+l^{*}$ & $l$ & $l^{*}$ & $l+l^{*}$ & $l$ & $l^{*}$ & $l+l^{*}$ & $\mathbf{p = 0}$ & $\mathbf{p = 0 . 5}$ & $\mathbf{p = 1}$ \\
\hline 109 & 218 & 31 & 167 & 198 & 75 & 95 & 170 & 100 & 74 & 174 & 5177 & 7125 & 7400 \\
\hline 310 & 620 & 44 & 503 & 547 & 186 & 319 & 505 & 280 & 163 & 443 & 22132 & 59334 & 45640 \\
\hline 535 & 1070 & 98 & 785 & 883 & 240 & 534 & 774 & 402 & 293 & 695 & 76930 & 128160 & 117786 \\
\hline 763 & 1526 & 144 & 1114 & 1258 & 385 & 780 & 1165 & 691 & 241 & 932 & 160416 & 300300 & 166531 \\
\hline 998 & 1996 & 83 & 1419 & 1502 & 425 & 862 & 1287 & 846 & 340 & 1186 & 117777 & 366350 & 287640 \\
\hline 1302 & 2604 & 134 & 2024 & 2158 & 704 & 1154 & 1858 & 1173 & 451 & 1624 & 271216 & 812416 & 529023 \\
\hline 1501 & 3002 & 119 & 2203 & 2322 & 784 & 1073 & 1857 & 1403 & 224 & 1627 & 262157 & 841232 & 314272 \\
\hline 1719 & 3438 & 131 & 2550 & 2681 & 856 & 1661 & 2517 & 1555 & 515 & 2070 & 334050 & 1421816 & 800825 \\
\hline 1990 & 3980 & 208 & 2339 & 2547 & 1013 & 1581 & 2594 & 1773 & 400 & 2173 & 486512 & 1601553 & 709200 \\
\hline 2159 & 4318 & 142 & 3238 & 3380 & 930 & 1816 & 2746 & 1823 & 445 & 2268 & 459796 & 1688880 & 811235 \\
\hline 2268 & 4536 & 148 & 3136 & 3284 & 952 & 1666 & 2618 & 1887 & 336 & 2223 & 464128 & 1586032 & 634032 \\
\hline 4323 & 8646 & 356 & 5852 & 6208 & 2238 & 3589 & 5827 & 3957 & 841 & 4798 & 2083312 & 8032182 & 3327837 \\
\hline
\end{tabular}



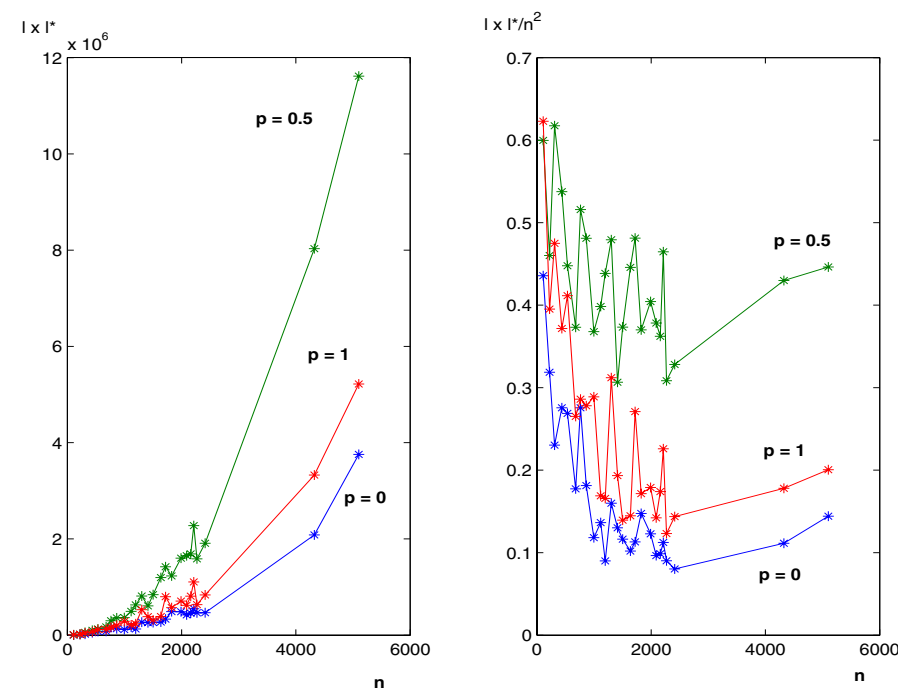

Fig. 6. Absolute (left) and normalized (divided by $n^{2}$ ) (right) results for visibility representation area requirement for different values of the parameter $p$ and triangulated planar graphs. The parameter $p=0$ (low longest path length $s t$-oriented graphs) is clearly preferable.

\subsection{Planar Graphs}

In this section we present some results for maximum density (triangulated) stplanar graphs. We also have similar results for low density planar graphs. We mainly present the impact of the parameter $p$ on the primal and dual longest path length of the planar graphs. From Table 2, it is clear that the primal and the dual longest path length are inversely proportional for various values of the parameter $p$. We have used the values $p=0,0.5,1$, as the most representative ones. The last three columns of Table 3 show the product $l \times l^{*}$. This is actually the area that is needed in order to construct a visibility representation of the given graph using the algorithms proposed in [2].
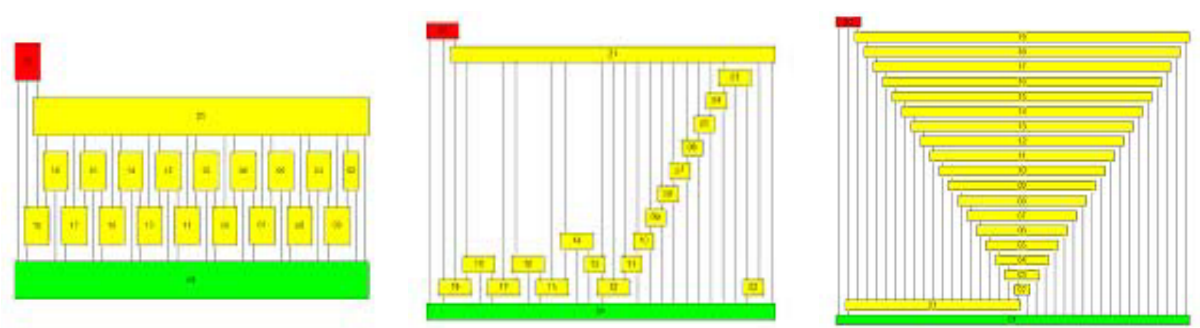

Fig. 7. Visibility Representations of a 21-path planar graph for different $s t$-orientations $(p=0,0.5,1)$ 

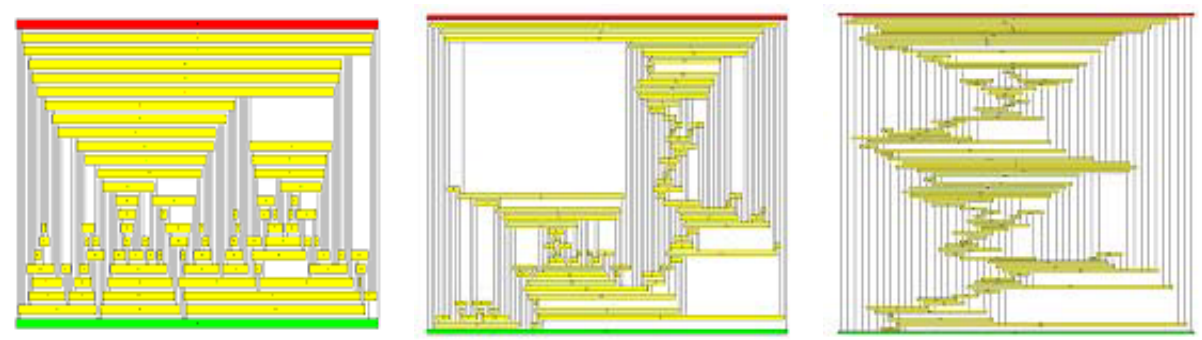

Fig. 8. Visibility Representations of a 85-node triangulated planar graph for different $s t$-orientations produced with $\operatorname{PAR}-\operatorname{STN}(p)(p=0,0.5,1)$
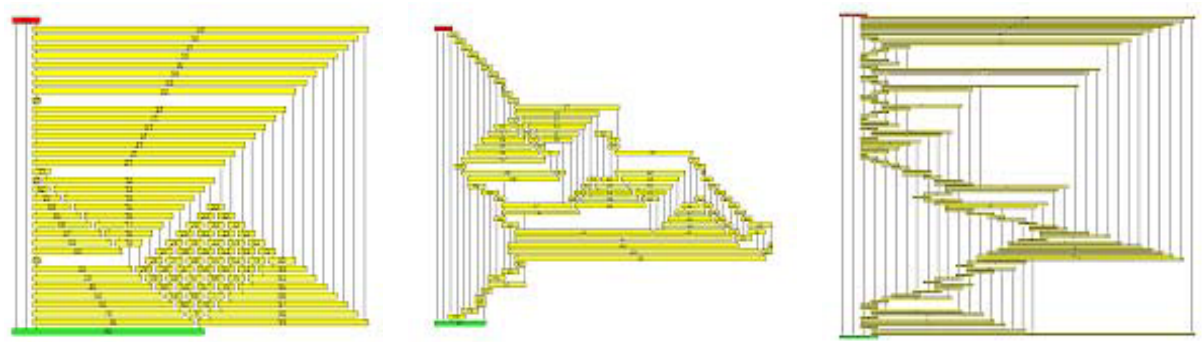

Fig. 9. Visibility Representations of a 10x10 grid graph for different st-orientations produced with PAR-STN $(p)(p=0,0.25,1)$

Figure 7 shows 3 visibility representation frames of a 21-path planar graph. The difference in the area is evident. Note that the visibility representation that uses the minimum st-orientation $(p=0)$ consumes the least area. Figure 8 contains 3 visibility representations frames of a triangulated graph where the value $p=0$ is preferable. Finally, in Figure 9 we present some visibility representations frames produced by st-orienting a grid graph. In this case, the importance of the parameter is clear. Using a parameterized st-orientation with $p=0.25$ is preferable, as it produces a more compact drawing.

\subsection{Orthogonal Drawings}

The impact of the different st-orientations is not very clear in orthogonal drawings. However, for the algorithm described in [3], where the area upper bound is roughly $0.76 n^{2}$, we are able to produce $s t$-numberings that produce drawings of area upper bound roughly equal to $0.68 n^{2}$ or less, by using the parameterized st-orientation algorithm. Due to space limitations, we cannot describe further details.

\section{Conclusions}

In this paper the application of parameterized st-orientations in graph drawing algorithms (mainly in visibility representations) is presented. It seems that there 
is a way to efficiently control the length of the longest path of an st-orientation and keep it "short", "long" or "medium". Experimental results not only on planar graphs but also on non-planar graphs reveal the robustness of the algorithm.

Acknowledgements. The authors would like to thank Hubert de Fraysseix, C.N.R.S. for his help on the visualization frames produced with his software P.I.G.A.L.E..

\section{References}

1. G.D. Battista, P. Eades, R. Tamassia, and I.G. Tollis. Graph Drawing: Algorithms for the Visualization of Graphs. Prentice Hall, 1999.

2. R. Tamassia and I.G. Tollis. A unified approach to visibility representations of planar graphs. Disc. and Comp. Geom., 1:321-341, 1986.

3. A. Papakostas and I.G. Tollis. Algorithms for area-efficient orthogonal drawings. Computational Geometry: Theory and Applications, 9:83-110, 1998.

4. S. Even and R. Tarjan. Computing an st-numbering. Theoretical Computer Science, 2:339-344, 1976.

5. A. Lempel, S. Even, and I. Cederbaum. An algorithm for planarity testing of graphs. In P. Rosestiehl(ed.) Theory of Graphs: International Symposium July 1966, pages 215-232, 1967.

6. J. Ebert. st-ordering the vertices of biconenected graphs. Computing, 30(1):19-33, 1983.

7. R. Tarjan. Two streamlined depth-first search algorithms. Fundamentae Informatica, 9:85-94, 1986.

8. P. Rosehnstiehl and R. Tarjan. Rectilinear planar layout and bipolar orientation of planar graphs. Discrete Comput. Geom., 1:343-353, 1986.

9. Y. Maon, B. Schieber, and U. Vishkin. Parallel ear decomposition search (eds) and st-numbering in graphs. Theoret. Comput. Sci, 47:277-298, 1986.

10. H.D. Fraysseix, P.O. de Mendez, and P. Rosenstiehl. Bipolar orientations revisited. Discrete Applied Mathematics, 56:157-179, 1995.

11. C. Papamanthou and I.G. Tollis. Algorithms for parameterized st-orientations of graphs, submitted to ESA2005.

12. J. Hopcroft and R. Tarjan. Efficient algorithms for graph manipulation. Comm. ACM, 16:372-378, 1973 .

13. J. Holm, K. de Lichtenberg, and M. Thorup. Poly-logarithmic deterministic fullydynamic algorithms for connectivity, minimum spanning tree, 2-edge and biconnectivity. J. ACM, 48(4):723-760, 2001.

14. Michael T. Goodrich and Roberto Tamassia. Data Structures and Algorithms in Java. John Wiley \& Sons, 2 edition, 2001. 